\title{
DETERMINANTES DEL GASTO EN EDUCACIÓN DE LOS HOGARES DE LA REGIÓN JUNÍN DEL PERU
}

\section{MAJOR FACTORS FOR EDUCATION EXPENDITURE FOR THE HOUSEHOLDS IN THE JUNIN REGION IN PERU}

\author{
Juan Lino Quispe ${ }^{8}$, Jesús Chávez Villarroe ${ }^{9}$
}

Facultad de Economía

\section{RESUMEN}

El estudio desarrolla un análisis descriptivo del gasto en educación al interior de los hogares, una comparación del mismo con el gasto público y la identificación de los factores que, en mayor 0 menor grado, determinan que las familias de la Región Junín inviertan más o menos en educación. Estos factores tienen que ver con el ingreso del hogar, la escolaridad del jefe de hogar, el número de miembros, el grado escolar que cursa el niño, el área geográfica y otros. Los resultados obtenidos permiten entender de mejor manera los mecanismos de decisión en los hogares a la hora de definir la cantidad de recursos que se destinarán a la educación de sus miembros.

Palabras clave: gasto, hogar, Ingreso, niño, jefe de hogar.

\section{ABSTRACT}

The study develops a descriptive analysis of education spending within households, a comparison of the spending in relation to others and the identification of factors that, in some degree, determine when families of the Junin Region invest more or less in education. These factors are related to household income, education of household head, number of members, the grade level of the children, geographic area and others. The results allow us to better understand the decision-making in homes in defining the amount of resources allocated in education of their members.

Key words: expenditure, household, income, child, children, head of household

\section{INTRODUCCIÓN}

La proporción del ingreso que las familias destinan a la educación de sus miembros, constituye un indicador del valor relativo que le asignan a la educación. Las familias deben enfrentarse a ciertos costos directos e indirectos, si su objetivo es incrementar el nivel educativo de sus miembros. Desde un punto de vista económico, las personas consideran la educación como una alternativa que les permite acrecentar sus posibilidades de incorporarse al mercado laboral en mejores condiciones y, por lo tanto, mejorar sus ingresos futuros. De este modo, la persona (o su familia) debe enfrentar los costos que representa educarse, y aceptar una reducción de los ingresos actuales que deja de percibir mientras estudia. Sin embargo, la decisión de educarse, y por tanto, la de gastar en educación, es un fenómeno más complejo, influido por factores sociales y culturales, además de los estrictamente económicos. Por tanto el estudio permite plantear como objetivo principal "la determinación y cuantificación de los principales factores que determinan el gasto en educación de los hogares en la Región Junín del Perú".

\footnotetext{
8 jlino55@yahoo.es

9 jchavez0702@hotmail.com
} 


\section{MATERIAL Y MÉTODOS}

Dada las características de no ser una investigación experimental, no se utilizan laboratorios ni talleres experimentales. Se hace uso de computadores y hojas de cálculo para el procesamiento de los datos. El método global para la realización del estudio se parte del problema, para luego ubicar el objeto y objetivo, lo que conlleva a determinar el campo de acción, esto permite la formulación de un modelo teórico nos lleva a la especificación de un modelo empírico y su respectiva aplicación.

\section{RESULTADOS Y DISCUSIÓN}

\section{Asistencia a las escuelas}

Según datos de la ENAHO-2009 existen diferencias importantes en la asistencia de los niños y la de las niñas en edad escolar, las mismas que responden a las características de su entorno. Por otro lado, el área geográfica de residencia de los hogares (urbana - rural) aparece como la variable que mayores diferencias presenta respecto a la asistencia, ya sea a escuelas públicas o privadas, con una diferencia de 8,3 puntos porcentuales entre áreas. Sin embargo, se debe considerar que las diferencias en la asistencia, al margen de los aspectos culturales y socioeconómicos, tienen que ver con problemas de insuficiencia en la oferta educativa latentes en comunidades rurales, tales como problemas de distancia, medios de comunicación y transporte, falta de infraestructura adecuada, carencia de maestros y otros, pudiendo generar problemas de abandono escolar e insuficiencia de cobertura educativa (Ministerio de Educación, 2009).

Tasas de asistencia escolar de la población de 5 a 17 años, según área geográfica, sexo

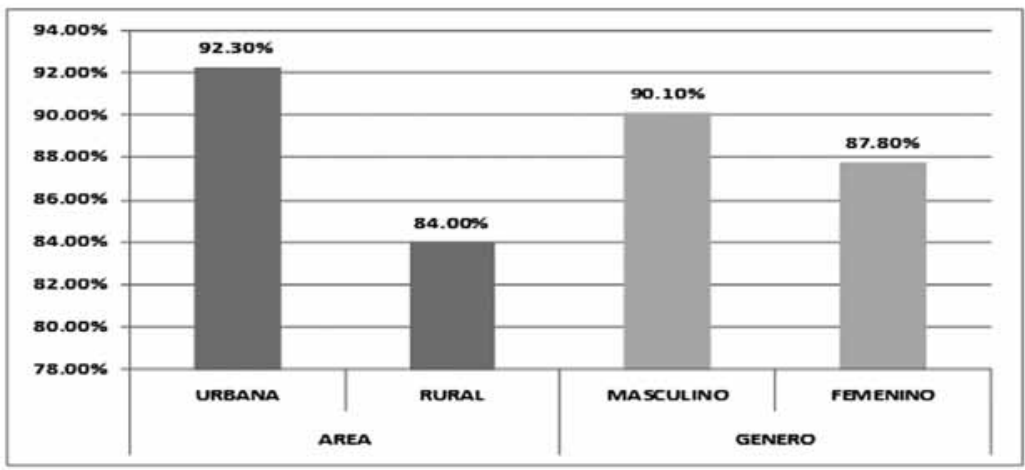

Fuente: Elaborado con información de la ENAHO-INEI

La asistencia de los niños está 2,3 puntos porcentuales por encima de las niñas, lo cual puede responder a un sesgo de preferencia de los padres en favor de los varones al momento de decidir quién debe o no estudiar.

Esta diferencia parece ser más alta en el área rural, ya que los hogares tienden a no matricular a las niñas por aspectos culturales, tales como la desconfianza en un sistema educativo adecuado (UNICEF, 2005) o sólo por preferir que permanezcan en casa para ayudar en las labores domésticas.

\section{Gasto público}

Nuestro país destina una proporción relativamente importante de recursos a educación (3.8\% del PIB el año 2005). Con este nivel de gasto, aunque se han alcanzado niveles razonablemente buenos 
en las tasas de matriculación neta de primaria, los niveles de culminación en primaria y más aún en secundaria, todavía son insuficientes; no obstante, están creciendo a un ritmo bastante favorable. El gasto público se financia con recursos internos y externos. La mayor parte se destina al pago de salarios del personal docente y administrativo de unidades educativas, institutos técnicos del nivel superior, institutos normales superiores, personal administrativo en el nivel distrital, departamental y nacional $(83,3 \%)$. La mayor parte del gasto corriente que no corresponde a salarios, se destina a la adquisición de materiales y suministros, siendo un componente importante el gasto en alimentación escolar, el cual es generalmente ejecutado por los gobiernos municipales, los que también destinan recursos al pago de servicios básicos (energía eléctrica, agua y teléfono). La otra parte importante corresponde al gasto de inversión ( $11,2 \%$ del gasto total).

\section{Gasto de los hogares}

El gasto que los hogares destinan a la educación depende de múltiples dimensiones, entre ellas, la más importante es el ingreso. El contexto del hogar determina las decisiones de enviar a los niños, niñas y adolescentes a la educación privada o a la educación pública. En la educación pública, el gasto de la familia es un complemento al gasto que realiza el Estado, vale decir, que tanto el Estado como la familia comparten el costo de educación de los niños, en tanto que en la educación privada, la totalidad del costo de la educación corre por cuenta del hogar. Además del contexto del hogar, la calidad de enseñanza es un factor que pesa a la hora de decidir el envío de los niños a una escuela privada.

Según se aprecia, existen hogares que reportaron que destinan recursos al pago de pensiones y matrícula, siendo que los hijos se encuentran asistiendo a un establecimiento público. Aunque no se cuenta con mayor evidencia, este hecho podría estar explicado por algunos cobros al margen de la norma, que estarían aplicando algunos establecimientos públicos para aceptar alumnos.

Estructura del gasto de los hogares en educación (\%)

\begin{tabular}{|l|r|r|r|}
\hline \multirow{2}{*}{\multicolumn{1}{c|}{ QUINTIL }} & \multicolumn{3}{c|}{ INGRESO PER CAPITA ANUAL } \\
\cline { 2 - 4 } & AÑ̃ 2007 & \multicolumn{1}{c|}{ AÑO 2008 } & AÑO 2009 \\
\hline QUINTIL 1 (MÁS POBRE) & 111.90 & 117.20 & 113.20 \\
\hline QUINTIL 2 & 244.20 & 223.60 & 214.80 \\
\hline QUINTIL 3 & 372.10 & 335.00 & 308.80 \\
\hline QUINTIL 4 & 522.80 & 489.60 & 448.20 \\
\hline QUINTIL 5 (MÁS RICO) & 961.00 & 966.30 & \multicolumn{1}{|c|}{896.00} \\
\hline NIVEL & \multicolumn{3}{|c|}{ SUBSIDIO MEDIO ANUAL } \\
\hline INICIAL & 59.60 & 60.50 & 56.90 \\
\hline PRIMARIA & 123.40 & 124.30 & 128.30 \\
\hline SECUNDARIA & 116.40 & 113.60 & 121.30 \\
\hline
\end{tabular}

Fuente: Elaborado con información de la ENAHO-INEI

Pese a que el Estado ha realizado esfuerzos en los últimos años por dotar de maestros e infraestructura suficiente al sistema educativo, en la actualidad, persisten altos niveles de déficit, principalmente de docentes, lo que lleva a que los padres en algunas escuelas, aporten dinero cada mes para cubrir el pago de un docente 0 para refaccionar infraestructura dañada. Estos aportes representarían alrededor de $0,6 \%$ y $0,8 \%$ del gasto en educación, respectivamente. 


\section{Prospectiva Universitaria}

El gasto en educación como parte del gasto total

De acuerdo a datos de la ENAHO-2009, los hogares del quintil más bajo disponen, en promedio, de S/160/mes por persona para cubrir las necesidades de cada miembro, cifra 8 veces menor que los recursos disponibles en los hogares que pertenecen al quintil superior (S/1331.50/mes). La disponibilidad de recursos en el hogar puede variar debido a distintos factores, como el número de miembros en el hogar, la educación de los padres y las remuneraciones en el mercado laboral, entre otros. En relación a los miembros en el hogar, un número grande de miembros no productivos (que no generen ingresos), en general, está asociado a una baja cantidad de recursos disponibles per cápita; en cambio, un número grande de miembros productivos se asocia a una mayor cantidad de recursos disponibles para todos los miembros del hogar.

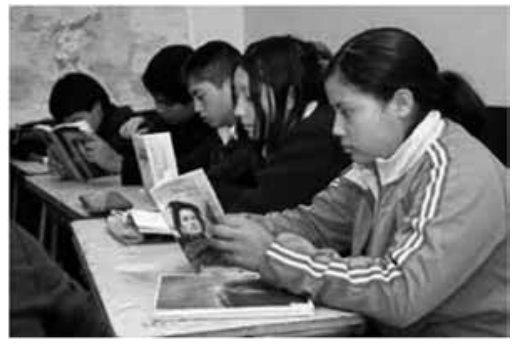

Distribución promedio del gasto mensual total (2009)

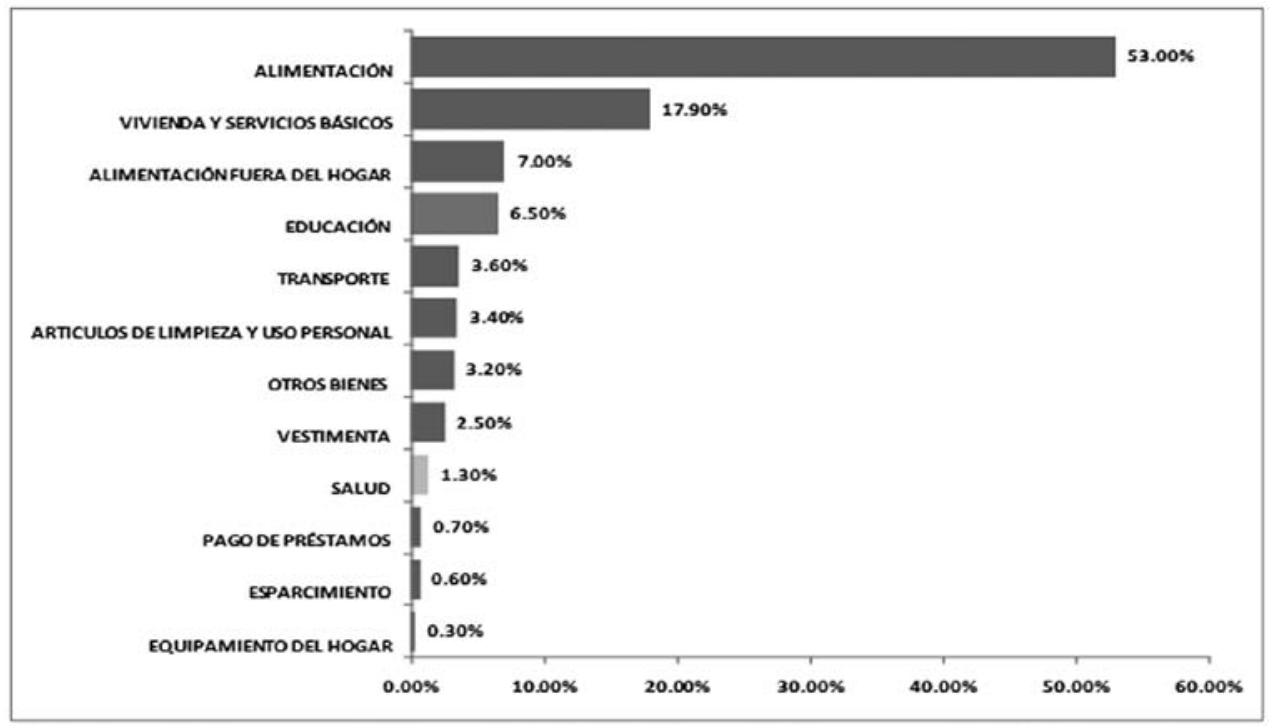

Fuente: Elaborado con información de la ENAHO-INEI

La información del gráfico anterior, permite tener mayor prioridad dentro del consumo. Sin embargo, una primera aproximación de la participación de este componente presenta mucha variación entre los distintos tipos de bienes en el presupuesto los diferentes hogares, lo que indicaría que su familiar. La característica más sobresaliente es importancia dentro del presupuesto se modifica la elevada proporción de recursos dirigidos a considerablemente según las características de alimentación (60\%), situándose como el bien de cada hogar. 
Estructura del gasto del hogar, según área geográfica (2009)

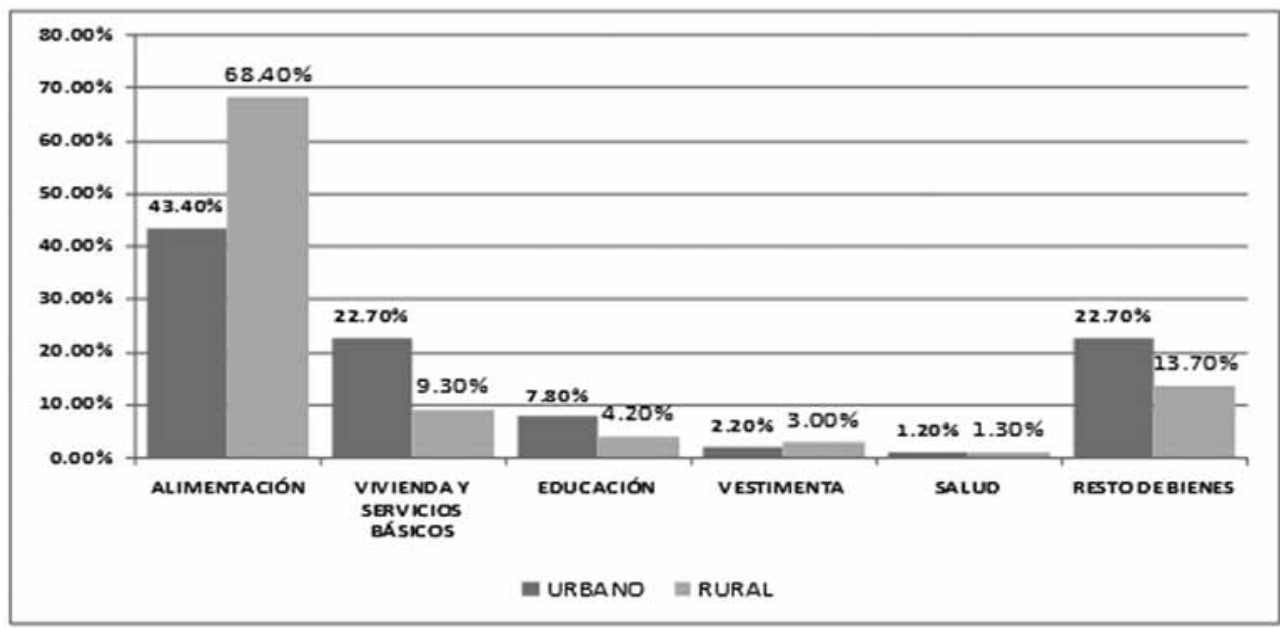

Fuente: Elaborado con información de la ENAHO-INEI

\section{DISCUSIÓN}

Estudiar los determinantes de la demanda de los servicios educativos y, específicamente, los factores que inciden en las decisiones de gasto en educación de los hogares, es un tema crucial en el análisis del bienestar de la niñez, en el sentido de que debe orientar la elaboración de políticas públicas hacia determinados grupos 0 segmentos de población.

\section{Variables que explican el gasto en educación}

Entre los principales factores se tiene:

- Percepciones personales y culturales

- Factores institucionales

- Factores económicos

- Factores socio demográficos

\section{Planteamiento del modelo}

Con base en las consideraciones anteriores, la ecuación a estimar queda especificada de la siguiente forma:

$$
\begin{aligned}
\log \left(\text { gast }_{\text {educ }}\right)=\alpha+ & \sum \beta \log (\text { ingresos })+\sum \chi \text { crac_hogar }+\sum \phi \text { carac_jefes } \\
& +\sum \gamma \text { ubic_geografica }+\sum \varphi \text { carac_niños }+\varepsilon
\end{aligned}
$$

\section{Hogares biparentales}

Los resultados de la estimación de la ecuación de gasto para los hogares biparentales se presentan en los cuadros posteriores. Los coeficientes estimados (elasticidades) para tres de las cinco variables de ingresos (ingreso laboral del jefe de hogar, ingreso no laboral del cónyuge e ingreso de los otros miembros del hogar), desde la óptica de las pruebas $t$, resultaron estadísticamente significativas al $1 \%$. De aquellas, el ingreso laboral del cónyuge, y el ingreso no laboral del jefe no resultaron estadísticamente significativos.

El efecto del ingreso no laboral del cónyuge $(0,050)$ es también importante, aunque menor que el efecto del ingreso laboral del jefe de hogar. Por otro lado, el hecho que el coeficiente estimado del ingreso no laboral de la esposa sea significativo proporciona pautas para suponer 
que las transferencias, subsidios u otros recursos no laborales percibidos por los cónyuges tienen un impacto positivo en el gasto de educación de los niños, a diferencia de si esas transferencias hubiesen sido entregadas al jefe de hogar (coeficiente no significativo).

Estimaciones del gasto en educación total por niño para familias biparentales (Variables significativas)

Variable dependiente: In(gasto en educación mensual total por niño)

\begin{tabular}{|c|c|c|c|c|c|}
\hline Caracteristicas & Variables & $\begin{array}{l}\text { Coeficientes } \\
\text { Estimados }\end{array}$ & SE & t-stat & Sig \\
\hline Constante & Constante & 6.058000 & 0.288000 & 21.034722 & 0.000000 \\
\hline \multirow{3}{*}{ Ingresos } & In(ingreso laboral JefeHogar) & 0.071000 & 0.014000 & 5.071429 & 0.000000 \\
\hline & In(ingreso no laboral JefeHogar) & 0.050000 & 0.015000 & 3.333333 & 0.000865 \\
\hline & In(ingreso total otros miembros) & 0.021000 & 0.008000 & 2.625000 & 0.008694 \\
\hline \multirow{2}{*}{ Caracteristicas del Hogar } & Energia Eléctrica & -0.212000 & 0.045000 & -4.711111 & 0.000003 \\
\hline & Número de niños en el hogar & -0.122000 & 0.011000 & -11.090909 & 0.000000 \\
\hline Ubicación Geográfica & Urbano-Rural & -0.345000 & 0.043000 & -8.023256 & 0.000000 \\
\hline \multirow{4}{*}{ Caracteristicas de los Niños } & Primaria & 0.865000 & 0.066000 & 13.106061 & 0.000000 \\
\hline & Secundaria & 1.293000 & 0.082000 & 15.768293 & 0.000000 \\
\hline & Establecimiento Público & -1.497000 & 0.084000 & -17.821429 & 0.000000 \\
\hline & Rezago & 0.255000 & 0.037000 & \begin{tabular}{|r|}
6.891892 \\
\end{tabular} & 0.000000 \\
\hline
\end{tabular}

Fuente: Elaborado con información de la ENAHO-INEI

En cuantoalas variables que caracterizan el hogar, la disponibilidad de energía eléctrica y el número de niños en el hogar resultaron significativos en el modelo, en tanto que el tipo del hogar no afecta como determinante para el gasto en educación. En este sentido, los resultados indican que los hogares con energía eléctrica gastan más en la educación de los niños que aquellos sin energía eléctrica pues, como se mencionó anteriormente, la disponibilidad de energía eléctrica es un reflejo de una mayor disponibilidad de ingresos en el hogar, lo que permite alcanzar un mayor gasto en educación.

En cuanto al número de niños en el hogar, los resultados indican que el gasto por niño en educación varía inversamente a la cantidad de niños presentes en el hogar, debido a que los recursos deben racionarse para cubrir el mayor número de necesidades. Sin embargo, esta reducción en el gasto por niño también puede justificarse por los menores costos de educación, en el sentido que algunos de los bienes necesarios en educación pueden ser utilizados o compartidos por todos los niños en el hogar (libros, uniformes, textos, entre otros). Asimismo, se observa que la variable de condición étnica del jefe de hogar no fue significativa, lo que sugiere que no existen diferencias significativas en niveles de gasto en educación entre hogares indígenas y no indígenas, cuando se controla por el resto de las variables.

Las características geográficas del hogar, identificadas por área de residencia (urbanorural), resultaron significativas en el modelo de negociación, lo que indica la existencia de una gran diferencia entre áreas geográficas en el gasto en educación. Según estos resultados, el gasto en el área rural es menor que en el área urbana en virtud del signo negativo del coeficiente estimado.

\section{Hogares mono parentales}

Para el caso de las familias mono parentales, la negociación se lleva a cabo sólo entre el jefe de 
hogar, pues el cónyuge está ausente, y los otros miembros. El modelo diferencia el efecto del ingreso laboral y no laboral del jefe de hogar y el ingreso total de los otros miembros. Según los resultados obtenidos, el jefe de hogar presenta similares elasticidades del gasto respecto al ingreso (laboral y no laboral). La elasticidad del ingreso de los otros miembros en el hogar tiene menor significancia estadística en la determinación del gasto de educación de los niños, que las elasticidades del ingreso, laboral y no laboral, del jefe de hogar. Estos resultados estarían indicando que los ingresos del jefe, como los de los otros miembros, están siendo destinados a la educación de los niños en el hogar. La presencia de sub núcleos familiares, además de otras relaciones en el hogar (hermanos, nietos, sobrinos), influiría dentro la negociación de inversión en educación de los hijos, fenómeno que no se observa en el análisis de hogares biparentales.

Estimaciones del gasto en educación total por niño para familias mono parentales (variables significativas)

Variable dependiente: In(gasto en educación mensual total por niño)

\begin{tabular}{|l|l|r|r|r|c|}
\hline \multicolumn{1}{|c|}{ Características } & \multicolumn{1}{|c|}{ Variables } & \multicolumn{1}{c|}{$\begin{array}{l}\text { Coeficientes } \\
\text { Estimados }\end{array}$} & \multicolumn{1}{c|}{ SE } & \multicolumn{1}{c|}{ t-stat } & Sig \\
\hline \multirow{2}{*}{ Constante } & Constante & 6.226000 & 0.544000 & 11.444853 & 0.000000 \\
\hline \multirow{3}{*}{ Ingresos } & In(ingreso laboral JefeHogar) & 0.036000 & 0.013000 & 2.769231 & 0.005642 \\
\cline { 2 - 6 } & In(ingreso no laboral JefeHogar) & 0.037000 & 0.013000 & 2.846154 & 0.004445 \\
\cline { 2 - 6 } & In(ingreso total otros miembros) & 0.025000 & 0.011000 & 2.272727 & 0.023089 \\
\hline \multirow{2}{*}{ Características del Hogar } & Energía Eléctrica & -0.372000 & 0.084000 & -4.428571 & 0.000010 \\
\cline { 2 - 6 } & Número de niños en el hogar & -0.134000 & 0.022000 & -6.090909 & 0.000000 \\
\hline \multirow{2}{*}{ Ubicación Geográfica } & Urbano-Rural & -0.372000 & 0.080000 & -4.650000 & 0.000003 \\
\hline \multirow{3}{*}{ Características de los Niños } & Primaria & 1.108000 & 0.204000 & 5.431373 & 0.000000 \\
\cline { 2 - 6 } & Secundaria & 1.524000 & 0.224000 & 6.803571 & 0.000000 \\
\cline { 2 - 6 } & Establecimiento Público & -1.497000 & 0.160000 & -9.356250 & 0.000000 \\
\cline { 2 - 6 } & Rezago & 0.144000 & 0.066000 & 2.181818 & 0.029174 \\
\hline
\end{tabular}

Fuente: Elaborado con información de la ENAHO-INEI

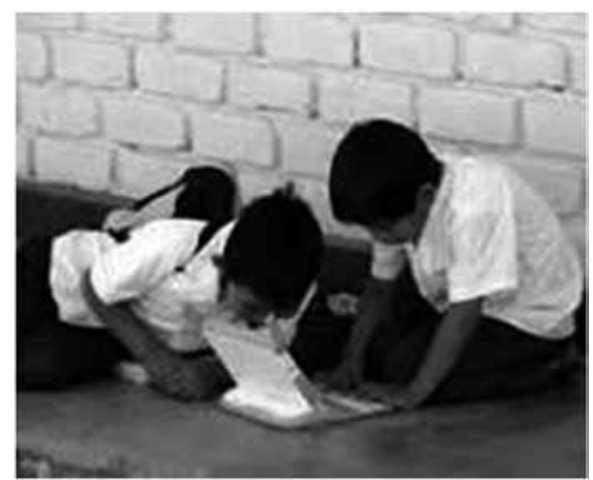

Asimismo, las estimaciones indican que ninguna de las características asociadas al jefe de hogar influye en la inversión en educación en los niños.
Sin embargo, la no existencia de efectos puede deberse a la importante presencia de hogares extendidos dentro los clasificados como mono parentales, ya que serían otros núcleos familiares, al interior del hogar, los que estarían influyendo en las decisiones de gasto en educación en el hogar.

Se ha analizado la participación de los hogares en el costo total que implica la educación de un niño, comparándola con el esfuerzo público. El costo de educar a un niño en el sistema privado, que alcanza en promedio a S/ 2.954 por año, es mayor al costo de hacerlo en la educación pública (S/ 1.741 por año, en promedio). Este hecho 
tendría que ver con el margen de utilidades que deben generar los establecimientos privados, y con el mayor gasto en la compra de textos, material escolar y ropa. Lo anterior sugiere que los niños de establecimientos privados disponen de más y mejores recursos pedagógicos y material de apoyo para sus estudios que los niños de establecimientos públicos.

Muchos padres optan por mantener a sus hijos en la educación privada, a pesar de que el gasto al que deben hacer frente es más alto en relación a la alternativa de la educación pública. La razón más importante tendría que ver con la mejor calidad de enseñanza que ofertan los establecimientos privados y la continuidad de labores durante la gestión. Estos factores inciden en un mejor desempeño escolar de los niños.

La estimación de las ecuaciones de gasto en educación por niño, para hogares biparentales y mono parentales, ha puesto en evidencia algunos resultados de interés, mismos que se detallan a continuación: Todo incremento en el ingreso disponible del hogar, trae consigo incrementos en el gasto destinado a la educación de los niños, tanto en hogares biparentales como mono parentales.

\section{REFERENCIAS BIBLIOGRÁFICAS}

Hernández, Julia. El capital humano en las teorías del crecimiento económico. España, Aportes, Revista de la Facultad de Economía, BUAP, Año XI, Número 33, Septiembre - Diciembre de 2006

Miranda, Liliana. Factores asociados al rendimiento escolar y sus implicancias para la política educativa del Perú. Lima: GRADE, 2008.

Vásquez, Enrique y Mendizábal, Enrique. Educación para una vida mejor. Lima: CIUP, Save the Children, 2002.

Vásquez, Enrique y Mendizábal, Enrique. Urgente: bienestar y justicia para la niñez más excluida. Lima: CIUP, Save the Children, 2002.

Yamada, Gustavo. Retornos a la educación superior en el mercado laboral: ¿vale la pena el esfuerzo. Lima: CIUP, CIES, 2007.

Tam, Mary. Eficiencia técnica del gasto en educación pública en las regiones del Perú. Lima: CIES, 2008. 